\title{
Neutrino Cross Sections at HERA and Beyond
}

\author{
Mary Hall Reno ${ }^{\text {a* }}$ \\ ${ }^{a}$ Department of Physics and Astronomy, University of Iowa, Iowa City, Iowa 52242 USA
}

The ultrahigh energy neutrino cross section is reviewed. Experimental results from HERA $e p$ and $e p$ scattering have yielded new information about the small $x$ behavior of the parton distribution functions. We compare pre-HERA era neutrino-nucleon cross sections with more recent evaluations.

\section{INTRODUCTION}

The sources of very high energy cosmic rays should also be sources of neutrinos. While the complete scheme of ultrahigh energy cosmic ray production is not yet understood [1, ancillary to cosmic ray production are neutrino fluxes, both at the source of the cosmic rays and through cosmic ray propagation and scattering with the microwave background. Given that neutrinos are unabsorbed and undeflected between their sources and the Earth, neutrino telescopes may yield results that allow for a better understanding of cosmic ray production 2]. The neutrinonucleon cross section is a key ingredient in neutrino telescope measurements.

The theoretical predictions of ultrahigh energy neutrino cross sections have been greatly influenced by the deep inelastic scattering (DIS) results of HERA experiments, starting in 1992. Neutrino cross sections were not measured at HERA, where beams of electrons and positrons are incident on proton beams, however, our knowledge of parton distribution functions has been dramatically improved with HERA results. At an energy equivalent to a neutrino energy of $54 \mathrm{TeV}$, the HERA results have aided our understanding of the parton distribution functions in new kinematic regimes and have led to different high energy extrapolations of the ultrahigh energy neutrino cross section than what was done earlier.

\footnotetext{
*To appear in the Proceedings of the XIII International Symposium on Very High Energy Cosmic Ray Interactions, 6-12 September 2004, Pylos, Greece.
}

We review the evaluation of the neutrinonucleon cross section and summarize the consequences of HERA results for the small $x$ region of the parton distribution functions, and therefore, for the ultrahigh energy neutrino cross section. Alternatives to straightforward QCD evolution of the parton distribution functions are reviewed and their consequences for the ultrahigh energy cross section are compared.

\section{NEUTRINO CROSS SECTION}

We begin with a review of the cross section for

$\nu_{\mu}(k) N(p) \rightarrow \mu\left(k^{\prime}\right) X$,

neutrino scattering with an isoscalar nucleon $N$. The charged current differential cross section, in terms of $x=Q^{2} /(2 p \cdot q), Q^{2}=-q^{2}, q=k-k^{\prime}$, $y=p \cdot q /(p \cdot k)$, and the nucleon mass $M$, is

$$
\begin{aligned}
\frac{d^{2} \sigma}{d x d y} & =\frac{2 G_{F}^{2} M E_{\nu}}{\pi\left(1+Q^{2} / M_{W}^{2}\right)^{2}} \\
& \times\left\{q\left(x, Q^{2}\right)+(1-y)^{2} \bar{q}\left(x, Q^{2}\right)\right\} .
\end{aligned}
$$

The parton distribution functions (PDFs) for the relevant quark and antiquark distributions are functions of $\left(x, Q^{2}\right)$ and labeled as $q\left(x, Q^{2}\right)$ and $\bar{q}\left(x, Q^{2}\right)$.

The structure of Eq. (2) together with a knowledge of the Dokshitzer-Gribov-Lipatov-AltarelliParisi (DGLAP) evolution [3] of the PDFs give a qualitative understanding of the energy behavior of the cross section. At low energies, where $Q^{2} \ll M_{W}^{2}$, the PDFs are nearly $Q^{2}$ independent, and the neutrino-nucleon cross section scales with incident neutrino energy $E_{\nu}$. At high energies, 
the increase in the PDFs with increasing $Q^{2}$ is more than offset by the decrease in the cross section due to the $W$-boson propagator. As a result, using the relation that

$x y\left(2 M E_{\nu}\right)=Q^{2}$,

and the approximation that $Q_{\max }^{2} \sim M_{W}^{2}$, one is led to the relation

$x \sim \frac{10^{4}}{\left(E_{\nu} / \mathrm{GeV}\right)}$.

At the highest energies of interest, on the order of $E_{\nu} \sim 10^{12} \mathrm{GeV}$, this translates to $x \sim$ $10^{-8}$. HERA measurements made a significant improvement in the range of $\left(x, Q^{2}\right)$ compared to fixed target measurements: at HERA energies, given that $y \leq 1, x \geq 10^{-5} \cdot Q^{2}$ is accessible. Information about small $x$ PDFs at values of $Q^{2} \sim 1 \mathrm{GeV}^{2}$ is then evolved to higher $Q^{2}$. For $Q^{2} \sim M_{W}^{2}$, one has only a limited range in $x$ directly measured at HERA [45]. The kinematic reach in $\left(x, Q^{2}\right)$ at HERA is bigger than for fixed target DIS, where e.g., for $E=350 \mathrm{GeV}$, $x>1.6 \times 10^{-3} \cdot Q^{2}$. In addition to DIS at HERA and in fixed target experiments, there are PDF measurements at colliders. Tevatron experimental results are limited to $x>10^{-3}$, however, these measurements are done at large $Q^{2}$ values $[\underline{6}$.

Parton distribution function parametrizations are extracted from fits to the world data from DIS and collider experiments. Details on the theory and experimental aspects of the extraction of PDFs can be obtained in Ref. [7, for example. A number of publications have provided PDF parametrizations, most recently in Refs. 8910, with prior versions which have evolved as more data were published. An interesting comparison is between the parton distribution functions which were one of the standards in the study of the physics of the proposed Superconducting Super Collider (SSC) by Eichten, Hinchliffe, Lane and Quigg (EHLQ) from 1984 [11, with a current PDF set such as the Pumplin et al.'s CTEQ6 fit [8. The $u, \bar{u}$ and gluon PDFs are shown in Figs. 1] and 2 for $Q^{2}=15 \mathrm{GeV}^{2}$ and for $Q^{2}=M_{W}^{2}$.

An important feature of the earlier fits was that at the reference value of $Q=Q_{0}$ at which the PDFs were parametrized, it was assumed that the sea distribution functions and gluon distribution function had small $x$ extrapolations following

$x f\left(x, Q^{2}\right) \sim$ constant .

This is in comparison to a steeper distribution at small $x$ for the modern set, where in Fig. 1 one sees a small $x$ behavior with

$x f\left(x, Q^{2}\right) \sim x^{-\lambda}$.

The small $x$ behavior at lower values of $Q$ has an influence on the extrapolation to even smaller $x$ values. It is to this subject that we now turn.

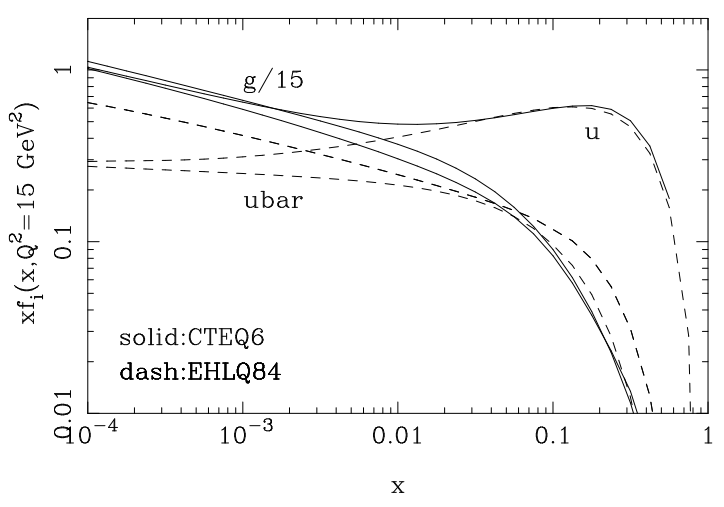

Figure 1. Up and $\bar{u}$ PDFs, and the gluon PDF rescaled by a factor of 15 , as a function of $x$ for $Q^{2}=15 \mathrm{GeV}^{2}$ for the EHLQ PDFs (dash) and CTEQ6 PDFs (solid).

\section{SMALL $x$ EXTRAPOLATIONS}

The small $x$ extrapolations of the PDFs in the DGLAP formalism can be inferred from the behavior of the gluon distribution. Small $x$ PDFs are important at ultrahigh energies where sea quarks dominate. This is manifest in Fig. 3 where the $\nu N$ and $\bar{\nu} N$ charged current cross sections are equal at $E \sim 10^{6} \mathrm{GeV}$. The sea distributions come from gluon splitting to quarkantiquark pairs,

$g \rightarrow q \bar{q}$, 


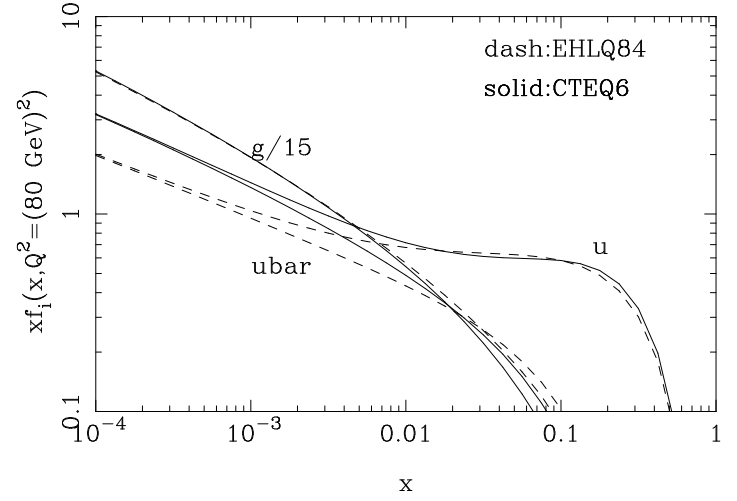

Figure 2. As in Fig. 1, now with $Q^{2}=M_{W}^{2}$.

so the gluon extrapolation at small $x$ is a good guide to the quark sea PDF extrapolations. For the typical values of $\lambda$ in modern parametrizations, if

$x g\left(x, Q_{0}^{2}\right) \sim A\left(Q_{0}^{2}\right) x^{-\lambda} \quad x \ll 1$,

the gluon distribution function has a similar form at higher values of $Q^{2}$ [12]:

$x g\left(x, Q^{2}\right) \sim A\left(Q^{2}\right) x^{-\lambda}$.

Our approach 13 has been to extrapolate the sea quarks in a similar way, matching the CTEQ6 PDFs below $x_{\min }=10^{-6}$ via

$x q\left(x, Q^{2}\right)=\left(\frac{x_{m i n}}{x}\right)^{\lambda} x q\left(x_{m i n}, Q^{2}\right)$.

The quantity $\lambda$ is determined for each flavor from the PDFs at $Q^{2}=M_{W}^{2}$. With the earlier EHLQ parametrizations, one would instead be lead to a small $x$ PDF extrapolation in the double-logarithmic-approximation (DLA) 14].

\section{CROSS SECTIONS}

It is the cross section evaluated using the EHLQ PDFs with a DLA extrapolation [15] that is shown in Fig. 3 by the dot-dashed line, while the dashed (for incident $\bar{\nu}$ 's) and solid (for incident $\nu$ 's) lines show the results using the power law extrapolation with $\lambda$. Similar results are obtained using the Gluck, Reya and Vogt 10 PDFs in the evaluation of the cross section 16. For reference, we also show the charged current cross section using the EHLQ PDFs with $Q^{2}$ frozen at $Q_{0}^{2}$.

An important reference point in Fig. [3 is at $E_{\nu} \sim 40 \mathrm{TeV}$, at which the neutrino interaction length is approximately equal to the diameter of the Earth. At higher energies, neutrinos traversing the Earth over trajectories through the Earth's core are significantly attenuated.

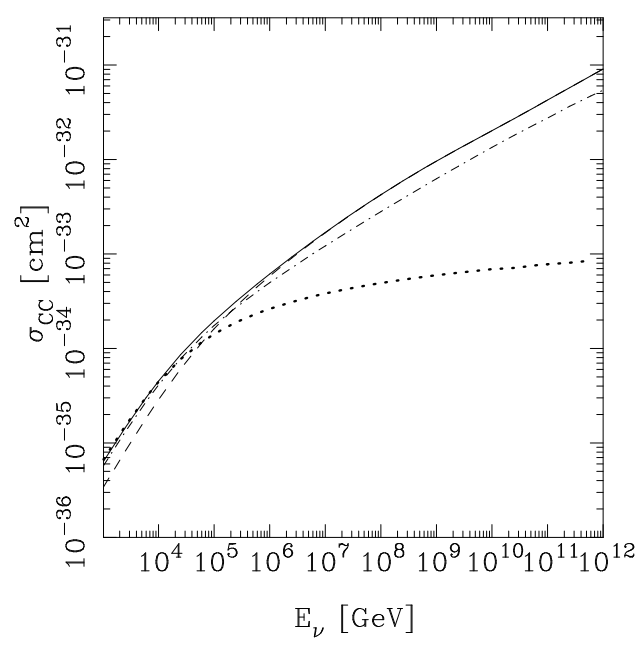

Figure 3. The charged current $\nu N$ (solid) and $\bar{\nu} N$ (dashed) cross sections as a function of incident neutrino/antineutrino energy, using the CTEQ6 PDFs. Also shown is the $\nu N$ cross section using the EHLQ PDFs (dot-dashed) and EHLQ PDFs frozen at the scale of $Q^{2}=5 \mathrm{GeV}^{2}$ (dotted).

The importance of QCD evolution in the context of neutrino scattering was first pointed out by Andreev, Berezinsky and Smirnov in Ref. [17. The curves in Fig. 3 make it clear that any additional modifications to the small $x$ PDFs must incorporate $Q^{2}$ evolution. One such modification is the inclusion of $\ln (1 / x)$ corrections using the Balitsky, Fadin, Kuraev and Lipatov (BFKL) formal- 
ism 18. Only a unified BFKL/DGLAP approach can be used for ultrahigh energy neutrino interactions. Kwiecinski, Martin and Stasto (KMS) have made such an evaluation 19. of the neutrino cross section, which is shown by the solid line in Fig. 4. Other theoretical work on the topic of $\ln (1 / x)$ corrections [20] cannot be applied directly to the neutrino nucleon cross sections until one has a form that explicitly includes DGLAP evolution.

Additional corrections have been made by $\mathrm{Ku}-$ tak and Kwiecinski (KK) in Ref. [21], where a nonlinear term is included in the evolution equations to account for gluon recombination effects. A continued growth of the small $x$ PDFs with decreasing $x$ ultimately will conflict with unitarity requirements. In parton language, the small $x$ PDFs will moderate due to gluon recombination:

$g g \rightarrow g$.

The KK result including recombination effects is shown in Fig. 4 by the dotted line. A different model for screening, the color dipole model of Golec-Biernat and Wusthoff (GBW) 22, as calculated in Ref. 21] is shown in the same figure with a dashed line. For reference, we also show the CTEQ6 result using the power law small $x$ extrapolation. All of the cross sections in Fig. 4 are in remarkably good agreement at $E_{\nu}=10^{12}$ $\mathrm{GeV}$.

Other references 2324] have recently appeared, including saturation or recombination effects. They are consistent with the range of results shown in Fig. 4.

\section{FINAL REMARKS}

Our knowledge of the parton distribution functions has greatly increased since HERA data became available in the early 1990's. Extrapolations based on DGLAP or a unified BFKL/DGLAP with and without recombination effects to $x$ values far below the measured regime at $Q^{2} \sim M_{W}^{2}$ all yield similar cross sections at $E_{\nu}=10^{12} \mathrm{GeV}$. There are prospects for new measurements of the PDFs with the upcoming Large Hadron Collider experiments, however, the smallest $x$ values relevant to ultrahigh energy neutrinos are outside our reach in collider experiments.

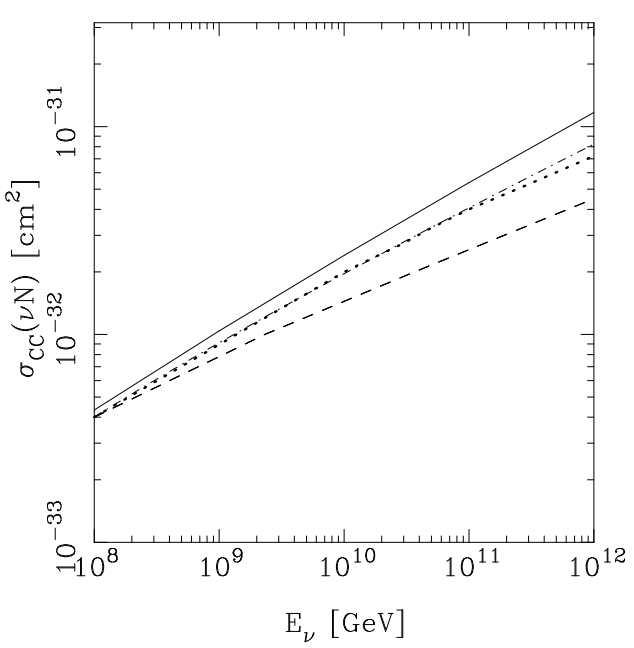

Figure 4. The charged current $\nu N$ cross section adapted from Ref. [21] of Kutak and Kwiecinski, showing $\sigma$ using a unified BFKL/DGLAP treatment with (dotted) and without (solid) screening. The CTEQ6 (dot-dash) result and a calculation using the GBW saturation model (dashed) from Ref. [21] are also shown.

The small $x$ behavior of the PDFs has implications in neutrino astrophysics for more than the neutrino-nucleon cross section. At lower energies, the small $x$ PDFs are inputs to $c \bar{c}$ and $b \bar{b}$ production by cosmic ray interactions with air nuclei. The semileptonic heavy quark decays are responsible for the prompt lepton fluxes which should dominate the atmospheric flux in the range of 100 $\mathrm{TeV}$ 2526]. Measurements of the prompt lepton flux may help constrain small $x$ physics.

\section{Acknowledgments}

The author thanks I. Sarcevic and I. Mocioiu for recent discussions and collaboration on the topic of the ultrahigh energy neutrino cross section, and I. Sarcevic, R. Gandhi and C. Quigg for their collaboration in years past. The work here was supported in part by U.S. D.O.E. contract DE-FG02-91ER40664. 


\section{REFERENCES}

1. See, e.g., R. J. Protheroe and R. W. Clay, Publ. Astron. Soc. Pac. 21 (2004) 1; P. Bhattacharjee and G. Sigl, Phys. Rept. 327 (2000) 109 and references therein.

2. J. G. Learned and K. Mannheim, Ann. Rev. Nucl. Part. Sci. 50 (2000) 679.

3. G. Altarelli and G. Parisi, Nucl Phys. B 126 (1977) 298; L. N. Lipatov, Sov. J. Nucl. Phys. 20 (1975) 95; V. N. Gribov and L. N. Lipatov, Sov. J. Nucl. Phys. 15 (1972) 438; Yu. L. Kokshitzer, Sov. Phys. JETP 46 (1977) 641. See also, e.g., G. Altarelli, Phys. Rep. 81 (1983).

4. C. Adloff et al. [H1 Collaboration], Eur. Phys. J. C 30(2003) 1.

5. S. Chekanov et al. [ZEUS Collaboration], Eur. Phys. J. C 32 (2003) 1; S. Chekanov et al. [ZEUS Collaboration], arXiv:hep-ex/0401003

6. See, e.g., Refs. [7-9] and references therein.

7. R. Devenish and A. Cooper-Sarkar, Deep Inelastic Scattering (Oxford University Press, Oxford, 2004).

8. J. Pumplin, D. R. Stump, J. Huston, H. L. Lai, P. Nadolsky and W. K. Tung, JHEP 0207 (2002) 012.

9. A. D. Martin, R. G. Roberts, W. J. Stirling and R. S. Thorne, Eur. Phys. J. C 28 (2003) 455; Eur. Phys. J. C 35 (2004) 325.

10. M. Gluck, E. Reya and A. Vogt, Eur. Phys. J. C 5 (1998) 461.

11. E. Eichten, I. Hinchliffe, K. D. Lane and C. Quigg, Rev. Mod. Phys. 56 (1984) 579 [Addendum-ibid. 58 (1986) 1065]. See also, D. W. Duke and J. F. Owens, Phys. Rev. D 30 (1984) 49.

12. R. K. Ellis, Z. Kunszt and E. M. Levin, Nucl. Phys. B 420 (1994) 517.

13. R. Gandhi, C. Quigg, M. H. Reno and I. Sarcevic, Phys. Rev. D 58 (1998) 093009. See also R. Gandhi, C. Quigg, M. H. Reno and I. Sarcevic, Astropart. Phys. 5(1996) 81; G. M. Frichter, D. W. McKay and J. P. Ralston, Phys. Rev. Lett. 74 (1995) 1508.

14. L. V. Gribov, E. M. Levin and M. G. Ryskin, Phys. Rep. 100 (1983) 1.

15. Earlier evaluations of the cross section, before the results of HERA experiments, appear in, e.g., D. W. McKay and J. P. Ralston, Phys. Lett. 167B (1986) 103; C. Quigg, M. H. Reno and T. P. Walker, Phys. Rev. Lett. 57 ( 1986) 774 .

16. M. Gluck, S. Kretzer and E. Reya, Astropart. Phys. 11 (1999) 327.

17. Yu. M. Andreev, V. S. Berezinsky and A. Yu. Smirnov, Phys. Lett. B 143 (1978) 521.

18. E. A. Kuraev, L. N. Lipatov and V. S. Fadin, Sov. Phys. JETP 45 (1977) 199 [Zh. Eksp. Teor. Fiz. 72 (1977) 377]; I. I. Balitsky and L. N. Lipatov, Sov. J. Nucl. Phys. 28 (1978) 822 [Yad. Fiz. 28 (1978) 1597].

19. J. Kwiecinski, A. Martin and A. Stasto, Phys. Rev. D 56 (1997) 3991.

20. G. Altarelli, R. D. Ball and S. Forte, Nucl. Phys. B 674 (2003) 459; M. Ciafaloni, D. Colferai, G. P. Salam and A. M. Stasto, Phys. Rev. D 68 (2003) 114003; M. Ciafaloni, D. Colferai, G. P. Salam and A. M. Stasto, Phys. Lett. B 587 (2004) 87; R. S. Thorne, Phys. Rev. D 64 (2001) 074005.

21. K. Kutak and J. Kwiecinski, Eur. Phys. J. C 29 (2003) 521.

22. K. Golec-Biernat and M. Wusthoff, Phys. Rev. D 59 (1999) 014017.

23. R. Fiore, L. L. Jenkovszky, A. Kotikov, F. Paccanoni, A. Papa and E. Predazzi, Phys. Rev. D 68 (2003) 093010.

24. M. V. T. Machado, Phys. Rev. D 70 (2004) 053008.

25. A. D. Martin, M. G. Ryskin and A. M. Stasto, Acta Phys. Polon. B 34 (2003) 3273.

26. For earlier work on prompt neutrinos and the gluon distribution function, see e.g., G. Gelmini, P. Gondolo and G. Varieschi, Phys. Rev. D 61 (2000) 056011; G. Gelmini, P. Gondolo and G. Varieschi, Phys. Rev. D 63 (2001) 036006; L. Pasquali, M. H. Reno and I. Sarcevic, Phys. Rev. D 59 (1999) 034020. 